\title{
Normal families of meromorphic functions sharing one function
}

\section{Ling Qiu and FeiFei Hu*}

\section{"Correspondence:}

hudiefei@emails.bjut.edu.cn

College of Applied Science, Beijing

University of Technology, Beijing,

100124, China

\begin{abstract}
Suppose $p(z)$ is a holomorphic function, the multiplicity of its zeros is at most $d, P(z)$ is a nonconstant polynomial. Let $\mathcal{F}$ be a family of meromorphic functions in a domain $D$, all of whose zeros and poles have multiplicity at least $\max \left\{\frac{k}{2}+d+1, k+d\right\}$. If for each pair of functions $f$ and $g$ in $\mathcal{F}, P(f) f^{(k)}$ and $P(g) g^{(k)}$ share a holomorphic function $p(z)$, then $\mathcal{F}$ is normal in $D$. It generalizes and extends the results of Jiang, Gao and $\mathrm{Wu}, \mathrm{Xu}$.
\end{abstract}

MSC: $30 \mathrm{D} 35 ; 30 \mathrm{D} 45$

Keywords: meromorphic function; normal family; shared holomorphic function

\section{Introduction and results}

Let $D$ be a domain in $\mathbb{C}$, let $\mathcal{F}$ be a family of meromorphic functions in $D . \mathcal{F}$ is said to be normal in $D$, in the sense of Montel, if for any sequence $\left\{f_{n}\right\} \in \mathcal{F}$ contains a subsequence $\left\{f_{n j}\right\}$ such that $f_{n j}$ converges spherically locally uniformly in $D$ to a meromorphic function or $\infty[1-3]$.

Let $a \in \mathbb{C} \cup\{\infty\}$, let $f$ and $g$ be two nonconstant meromorphic functions in $D$. If $f(z)-a$ and $g(z)-a$ have the same zeros (ignoring multiplicity), we say $f$ and $g$ share the value $a$ in $D$.

In 1959, Hayman [1] proved that if $f$ is a transcendental meromorphic function, then $f^{n} f^{\prime}$ assumes every finite nonzero complex number infinitely often for any positive integer $n \geq 3$. He [4] conjectured that this remains valid for $n=1$ and $n=2$. Further, the case of $n=2$ was confirmed by Mues [5] in 1979. The case $n=1$ was considered and settled by Clunie [6].

In 1994, Yang and Yang [7] proposed a conjecture: If $f$ is an entire function and $k \geq 2$, then $\left(f^{(k)}\right)^{n}-a(z)(a(z) \not \equiv 0)$ has infinitely many zeros.

Zhang and Song [8] proved the following theorem.

Theorem A Suppose that $f$ is a transcendental meromorphic function, $n, k$ are two positive integers, then when $n \geq 2,\left(f f^{(k)}\right)^{n}-a(z)$ has infinitely many zeros, where $a(z) \not \equiv 0$ is a small function of $f$.

In 2005, Wang [9] proved the following theorem. 
Theorem B Let $f$ be a transcendental meromorphic function, let $c(z) \not \equiv 0$ be a small function of $f$, and let $n, k$ be two positive integers. If $n \geq 3$, then $f^{n} f^{(k)}-c(z)$ has infinitely many zeros.

In the case of $f f^{(k)}$, Yang and Yang [7] proposed a conjecture: If $f$ is transcendental, then $f^{(k)}$ assumes every finite nonzero complex number infinitely often. In 2006, Wang [10] proved that this conjecture holds when $f$ has only zeros of multiplicity at least $k+1(k \geq 2)$.

In 2011, Meng and $\mathrm{Hu}$ [11] obtained the following theorem.

Theorem $\mathrm{C}$ Take a positive integer $k$ and a nonzero complex number $a$. Let $\mathcal{F}$ be a family of meromorphic functions in a domain $D \in \mathbb{C}$ such that each $f \in \mathcal{F}$ has only zeros of multiplicity at least $k+1$. For each pair $(f, g) \in \mathcal{F}$, ifff $f^{(k)}$ and $g g^{(k)}$ share $a$, then $\mathcal{F}$ is normal in $D$.

In 2011, Jiang and Gao [12] obtained the following theorem.

Theorem D Suppose that $d(\geq 0)$ is an integer, $p(z)$ is an analytic function in $D$, and the multiplicity of its all zeros is at most $d$. Let $\mathcal{F}$ be a family of meromorphic functions in $D$, let $n$ be a positive integer. If $n \geq 2 d+2$ and for each pair of functions $f$ and $g$ in $\mathcal{F}, f^{n} f^{\prime}$ and $g^{n} g^{\prime}$ share $p(z)$ in $D$, then $\mathcal{F}$ is normal in $D$.

In 2012, Wu and Xu [13] got the following theorem.

Theorem $\mathbf{E}$ Let $k$ be a positive integer, let $b \neq 0$ be a finite complex number, let $P$ be $a$ polynomial with either $\operatorname{deg} P \geq 3$ or $\operatorname{deg} P=2$ and $P$ having only one distinct zero, and let $\mathcal{F}$ be a family of meromorphic functions in $D$, all of whose zeros have multiplicity at least $k$. Iffor each pair offunctions $f$ and $g$ in $\mathcal{F}, P(f) f^{(k)}$ and $P(g) g^{(k)}$ share b in $D$, then $\mathcal{F}$ is normal in $D$.

It is natural to ask whether Theorem $\mathrm{E}$ can be improved by the idea of sharing a holomorphic function. In this paper, we study the problem and obtain the following theorems.

Theorem 1.1 Suppose that $d \geq 0$ is an integer, $p(z) \not \equiv 0$ is a holomorphic function in $D$, and the multiplicity of its all zeros is at most $d$. Let $\mathcal{F}$ be a family of meromorphic functions in $D$, the multiplicity of all zeros and poles of $f \in \mathcal{F}$ is at least $\max \left\{\frac{k}{2}+d+1, k+d\right\}$. If for each pair of functions $f$ and $g$ in $\mathcal{F}, f^{(k)}$ and $g g^{(k)}$ share $p(z)$ in $D$, then $\mathcal{F}$ is normal in $D$.

Remark 1.1 Theorem 1.1 still holds when $p(z)$ is a nonzero finite constant.

Theorem 1.2 Suppose that $d \geq 0$ is an integer, $p(z) \not \equiv 0$ is a holomorphic function in $D$, and the multiplicity of its all zeros is at most $d$. Let $P$ be a nonconstant polynomial, $\mathcal{F}$ be a family of meromorphic functions in $D$, the multiplicity of all zeros and poles of $f \in \mathcal{F}$ is at least $\max \left\{\frac{k}{2}+d+1, k+d\right\}$. If for each pair of functions $f$ and $g$ in $\mathcal{F}, P(f) f^{(k)}$ and $P(g) g^{(k)}$ share $p(z)$ in $D$, then $\mathcal{F}$ is normal in $D$.

\section{Some lemmas}

Lemma 2.1 (see [14]) Let $k$ be a positive integer, let $\mathcal{F}$ be a family of meromorphic functions in $D$ such that each function $f \in \mathcal{F}$ has only zeros with multiplicities at least $k$, and suppose 
that there exists $A \geq 1$ such that $\left|f^{(k)}(z)\right| \leq A$ whenever $f(z)=0, f \in \mathcal{F}$. If $\mathcal{F}$ is not normal at $z_{0} \in D$, then for each $\alpha, 0 \leq \alpha \leq k$, there exists a sequence of complex numbers $z_{n} \in D$, $z_{n} \rightarrow z_{0}$, a sequence of positive numbers $\rho_{n} \rightarrow 0$, and a sequence of functions $f_{n} \in \mathcal{F}$ such that

$$
g_{n}(\xi)=\frac{f_{n}\left(z_{n}+\rho_{n} \xi\right)}{\rho_{n}^{\alpha}} \rightarrow g(\xi)
$$

locally uniformly with respect to the spherical metric, where $g$ is a nonconstant meromorphic function on $\mathbb{C}$, all of whose zeros have multiplicity at least $k$, such that $g^{\sharp}(\xi) \leq g^{\sharp}(0)=$ $k A+1$. Moreover, $g(\xi)$ has order at most 2 .

Lemma 2.2 (see [15]) Let $f(z)$ be a meromorphic function and $k$ be a positive integer. If $f^{(k)} \not \equiv 0$, then

$$
N\left(r, \frac{1}{f^{(k)}}\right) \leq N\left(r, \frac{1}{f}\right)+k \bar{N}(r, f)+S(r, f) .
$$

Lemma 2.3 (see [1]) Let $f_{1}(z), f_{2}(z)$ be two meromorphic functions defined in $D=\{z:|z|<$ $R\}$, then

$$
N\left(r, f_{1} f_{2}\right)-N\left(r, \frac{1}{f_{1} f_{2}}\right)=N\left(r, f_{1}\right)+N\left(r, f_{2}\right)-N\left(r, \frac{1}{f_{1}}\right)-N\left(r, \frac{1}{f_{2}}\right) .
$$

Lemma 2.4 (see [16]) Let $f$ be a transcendental meromorphic function, let $P_{f}(z), Q_{f}(z)$ be two differential polynomials off. If $f^{n} P_{f}=Q_{f}$ holds and the degree of $Q_{f}$ is at most $n$, then $m\left(r, P_{f}\right)=S(r, f)$.

Lemma 2.5 Let $d(\geq 0)$ be an integer, let $k$ be a positive integer, and let $p(z)=a_{d} z^{d}+$ $a_{d-1} z^{d-1}+\cdots+a_{1} z+a_{0}$ be a polynomial, where $a_{d} \neq 0, a_{d-1}, \ldots, a_{0}$ are constants. Suppose that $f$ is a transcendental meromorphic function, all of whose zeros and poles have multiplicity at least $2, p(z)$ is a small function of $f(z)$, then $f^{(k)}(z)-p(z)$ has infinitely many zeros.

Proof Let

$$
\psi(z)=f^{(k)}-p(z)
$$

Suppose $f^{(k)}-p(z)$ has only finitely many zeros, then $N\left(r, \frac{1}{\psi(z)}\right)=S(r, f)$. By $(2.1)$, then

$$
\left(\frac{\psi}{p}\right)^{\prime}=\frac{f^{\prime} f^{(k)}}{p}+\frac{f^{(k+1)}}{p}+\left(\frac{1}{p}\right)^{\prime} f^{(k)} .
$$

Let

$$
\psi_{1}=\frac{\psi}{p}
$$

Since the multiplicity of zeros of $f(z)$ is at least 2 , we can get from (2.2) that

$$
N\left(r, \frac{1}{f}\right) \leq N\left(r, \frac{1}{\left(\psi_{1}\right)^{\prime}}\right)+S(r, f)
$$


By Lemma 2.2, we know that

$$
N\left(r, \frac{1}{\left(\psi_{1}\right)^{\prime}}\right) \leq N\left(r, \frac{1}{\psi_{1}}\right)+\bar{N}(r, f)+S(r, f) .
$$

We can get from (2.2) that

$$
\frac{f^{(k)}}{p} \frac{\left(\psi_{1}\right)^{\prime}}{\psi_{1}}-\frac{f^{\prime} f^{(k)}}{p}-\frac{f^{(k+1)}}{p}-\left(\frac{1}{p}\right)^{\prime} f^{(k)}=\frac{\left(\psi_{1}\right)^{\prime}}{\psi_{1}}
$$

i.e.,

$$
f\left(\frac{f^{(k)}}{p} \frac{\left(\psi_{1}\right)^{\prime}}{\psi_{1}}-\frac{f^{\prime} f^{(k)}}{f p}-\frac{f^{(k+1)}}{p}-\left(\frac{1}{p}\right)^{\prime} f^{(k)}\right)=\frac{\left(\psi_{1}\right)^{\prime}}{\psi_{1}}
$$

Let

$$
f H=\frac{\left(\psi_{1}\right)^{\prime}}{\psi_{1}}
$$

where $H=\frac{f^{(k)}}{p} \frac{\left(\psi_{1}\right)^{\prime}}{\psi_{1}}-\frac{f^{\prime} f^{(k)}}{f p}-\frac{f^{(k+1)}}{p}-\left(\frac{1}{p}\right)^{\prime} f^{(k)}$. By Lemma 2.4, we get $m(r, H)=S(r, f)$.

From (2.5) and Lemma 2.3, we obtain that

$$
\begin{aligned}
m\left(r, \frac{1}{f}\right) & \leq m(r, H)+m\left(r, \frac{\psi_{1}}{\left(\psi_{1}\right)^{\prime}}\right) \\
& \leq N\left(r, \frac{\left(\psi_{1}\right)^{\prime}}{\psi_{1}}\right)-N\left(r, \frac{\psi_{1}}{\left(\psi_{1}\right)^{\prime}}\right)+m\left(r, \frac{\left(\psi_{1}\right)^{\prime}}{\psi_{1}}\right)+S(r, f) \\
& \leq N\left(r,\left(\psi_{1}\right)^{\prime}\right)+N\left(r, \frac{1}{\psi_{1}}\right)-N\left(r, \frac{1}{\left(\psi_{1}\right)^{\prime}}\right)-N\left(r, \psi_{1}\right)+S(r, f) \\
& \leq \bar{N}(r, f)+N\left(r, \frac{1}{\psi}\right)-N\left(r, \frac{1}{\left(\psi_{1}\right)^{\prime}}\right)+S(r, f) .
\end{aligned}
$$

We can get from (2.6) that

$$
f^{(k)}\left(\frac{\left(\psi_{1}\right)^{\prime}}{p \psi_{1}}-\frac{f^{\prime}}{f p}-\frac{f^{(k+1)}}{p f^{(k)}}-\left(\frac{1}{p}\right)^{\prime}\right)=\frac{\left(\psi_{1}\right)^{\prime}}{\psi_{1}} .
$$

Let

$$
f^{(k)} G=\frac{\left(\psi_{1}\right)^{\prime}}{\psi_{1}}
$$

where $G=\frac{\left(\psi_{1}\right)^{\prime}}{p \psi_{1}}-\frac{f^{\prime}}{f p}-\frac{f^{(k+1)}}{p f^{(k)}}-\left(\frac{1}{p}\right)^{\prime}$. By Lemma 2.4, then $m(r, G)=S(r, f)$.

By (2.9), we have that

$$
\begin{aligned}
m\left(r, f^{(k)}\right) & \leq m\left(r, \frac{\left(\psi_{1}\right)^{\prime}}{\psi_{1}}\right)+m\left(r, \frac{1}{f}\right)+m\left(r, \frac{1}{G}\right) \\
& \leq m\left(r, \frac{1}{f}\right)+N(r, G)-N\left(r, \frac{1}{G}\right)+S(r, f)
\end{aligned}
$$


Since $\frac{\left(\psi_{1}\right)^{\prime}}{\psi_{1}}$ has only simple poles, and by (2.9) we know that the poles of $f$ are impossible $G$ 's. Hence the poles of $G$ are only possible from the zeros and poles of $p(z)$ or the zeros of $\psi_{1}, f$ and $f^{(k)}$.

Hence by (2.8) and (2.9), we obtain that

$$
\begin{aligned}
N(r, G) & \leq \bar{N}\left(r, \frac{1}{\psi_{1}}\right)+\bar{N}\left(r, \frac{1}{f}\right)+\bar{N}\left(r, \frac{1}{f^{(k)}}\right)+N\left(r, \frac{1}{p}\right) \\
& \leq \bar{N}\left(r, \frac{1}{\psi}\right)+\bar{N}\left(r, \frac{1}{f}\right)+\bar{N}\left(r, \frac{1}{f^{(k)}}\right)+S(r, f) .
\end{aligned}
$$

Since $\frac{\left(\psi_{1}\right)^{\prime}}{\psi_{1}}$ has only simple poles, so by $(2.9)$ we know that

$$
N\left(r, \frac{1}{G}\right) \geq N(r, f)+N\left(r, f^{(k)}\right)-\bar{N}(r, f) .
$$

Combining (2.7) and (2.10)-(2.12), we have

$$
\begin{aligned}
m\left(r, f^{(k)}\right) \leq & \left\{\bar{N}\left(r, \frac{1}{\psi}\right)+\bar{N}\left(r, \frac{1}{f}\right)+\bar{N}\left(r, \frac{1}{f^{(k)}}\right)\right\}-\left\{N(r, f)+N\left(r, f^{(k)}\right)-\bar{N}(r, f)\right\} \\
& +\left\{\bar{N}(r, f)+N\left(r, \frac{1}{\psi}\right)-N\left(r, \frac{1}{\left(\psi_{1}\right)^{\prime}}\right)\right\}+S(r, f) .
\end{aligned}
$$

Hence

$$
\begin{aligned}
T\left(r, f^{(k)}\right) \leq & \bar{N}\left(r, \frac{1}{\psi}\right)+\bar{N}\left(r, \frac{1}{f}\right)+\bar{N}\left(r, \frac{1}{f^{(k)}}\right)-N(r, f)+\bar{N}(r, f) \\
& +\bar{N}(r, f)+N\left(r, \frac{1}{\psi}\right)-N\left(r, \frac{1}{\left(\psi_{1}\right)^{\prime}}\right)+S(r, f) .
\end{aligned}
$$

Since the multiplicity of the zeros and poles of $f(z)$ is at least 2, by an elementary calculation and combing with Lemma 2.2, (2.3) and (2.4), the above inequality yields

$$
\begin{aligned}
T\left(r, f^{(k)}\right) & \leq N\left(r, \frac{1}{f^{(k)}}\right)+2 N\left(r, \frac{1}{\psi}\right)+S(r, f) \\
& \leq N\left(r, \frac{1}{f}\right)+k \bar{N}(r, f)+2 N\left(r, \frac{1}{\psi}\right)+S(r, f) \\
& \leq(k+1) \bar{N}(r, f)+3 N\left(r, \frac{1}{\psi}\right)+S(r, f) .
\end{aligned}
$$

Since the multiplicity of the poles of $f(z)$ is at least 2, we can get from (2.13) that

$$
\begin{aligned}
T\left(r, f^{(k)}\right) & \leq\left(1-\frac{1}{k+2}\right) N\left(r, f^{(k)}\right)+3 N\left(r, \frac{1}{\psi}\right)+S(r, f) \\
& \leq\left(1-\frac{1}{k+2}\right) N\left(r, f^{(k)}\right)+S(r, f) .
\end{aligned}
$$

This implies $T\left(r, f^{(k)}\right)=S(r, f)$, then $f^{(k)}$ is a rational function, thus $f$ is a rational function which contradicts with $f$ is transcendental. Hence $f^{(k)}(z)-p(z)$ has infinitely many zeros. 
Remark 2.1 When $p(z)$ is a nonzero finite constant or a small function of $f(z)$, similarly we can get the same conclusion.

Lemma 2.6 Let $d(\geq 0)$ be an integer, let $k$ be a positive integer, and let $p(z)=a_{d} z^{d}+$ $a_{d-1} z^{d-1}+\cdots+a_{1} z+a_{0}$ be a polynomial, where $a_{d} \neq 0, a_{d-1}, \ldots, a_{0}$ are constants. Iff $(z)$ is a nonconstant polynomial, all of whose zeros and poles have multiplicity at least $k+d$, then $f^{(k)}(z)-p(z)$ has at least two distinct zeros, and $f f^{(k)}(z)-p(z) \not \equiv 0$.

Proof We discuss the following two cases.

Case 1. If $f f^{(k)}-p(z) \neq 0$, then $f f^{(k)}-p(z) \equiv C$, where $C$ is a nonzero constant. So $f^{(k)} \equiv$ $p(z)+C$. Since the multiplicity of all the zeros of $f$ is at least $k+d$, thus $\operatorname{deg}\left(f f^{(k)}\right) \geq k+2 d$, which contradicts with $\operatorname{deg}(p(z))=d$.

Case 2. If $f f^{(k)}-p(z)$ has only one zero $z_{0}$, we assume $f^{(k)}-p(z) \equiv A\left(z-z_{0}\right)^{l}$, where $A$ is a nonzero constant, $l$ is a positive integer.

We discuss the following two cases.

(i) If $l \leq d+1$, then $f f^{(k)} \equiv p(z)+A\left(z-z_{0}\right)^{l}$. Since $\operatorname{deg}\left(f f^{(k)}\right) \geq k+2 d$, the degree of the right of the equation is at most $d+1$, which is smaller than the degree of the left of the equation. We get a contradiction.

(ii) If $l>d+1$, then $f f^{(k)} \equiv p(z)+A\left(z-z_{0}\right)^{l}$. So $\left(f f^{(k)}\right)^{(d)} \equiv a_{d}+A l \cdots(l-d+1)\left(z-z_{0}\right)^{l-d}$. Since $a_{d} \neq 0$, so $\left(f^{(k)}\right)^{(d)}$ has only simple zeros, which contradicts with the multiplicity of all the zeros of $f$ is at least $k+d$.

By Case 1 and Case $2, f^{(k)}-p(z)$ has at least two distinct zeros.

If $f f^{(k)}-p(z) \equiv 0$, then similar to the proof of Case 1 , we get a contradiction. Hence $f^{(k)}-p(z) \not \equiv 0$.

Lemma 2.7 Let $d(\geq 0)$ be an integer, let $k$ be a positive integer, and let $p(z)=a_{d} z^{d}+$ $a_{d-1} z^{d-1}+\cdots+a_{1} z+a_{0}$ be a polynomial, where $a_{d} \neq 0, a_{d-1}, \ldots, a_{0}$ are constants. If $f(z)$ is a nonconstant rational function and not a polynomial, and the multiplicity of whose zeros and poles is at least $\frac{k}{2}+d+1$, then $f^{(k)}(z)-p(z)$ has at least two distinct zeros, and $f^{(k)}(z)-p(z) \not \equiv 0$.

Proof Since $f(z)$ is a nonconstant rational function and not a polynomial, then obviously $f^{(k)}(z)-p(z) \not \equiv 0$. Let

$$
f(z)=B \frac{\left(z-\alpha_{1}\right)^{m_{1}}\left(z-\alpha_{2}\right)^{m_{2}} \cdots\left(z-\alpha_{s}\right)^{m_{s}}}{\left(z-\beta_{1}\right)^{n_{1}}\left(z-\beta_{2}\right)^{n_{2}} \cdots\left(z-\beta_{t}\right)^{n_{t}}}
$$

where $B$ is a nonzero constant. Since the multiplicity of the zeros and poles of $f$ is at least $\frac{k}{2}+d+1$, we have $m_{i} \geq \frac{k}{2}+d+1(i=1,2, \ldots, s), n_{j} \geq \frac{k}{2}+d+1(j=1,2, \ldots, t)$. For simplicity, we denote

$$
m_{1}+m_{2}+\cdots+m_{s}=m \geq\left(\frac{k}{2}+d+1\right) s, \quad n_{1}+n_{2}+\cdots+n_{t}=n \geq\left(\frac{k}{2}+d+1\right) t .
$$

By (2.19), we get

$$
f^{(k)}(z)=B \frac{\left(z-\alpha_{1}\right)^{m_{1}-k}\left(z-\alpha_{2}\right)^{m_{2}-k} \cdots\left(z-\alpha_{s}\right)^{m_{s}-k} g(z)}{\left(z-\beta_{1}\right)^{n_{1}+k}\left(z-\beta_{2}\right)^{n_{2}+k} \cdots\left(z-\beta_{t}\right)^{n_{t}+k}},
$$


where $g(z)=(m-n)(m-n-1) \cdots(m-n-k+1) z^{k(s+t-1)}+\cdots+c_{1} z+c_{0}$ is a polynomial, $c_{i}$ $(i=0,1)$ are constants and $\operatorname{deg}(g(z)) \leq k(s+t-1)$. Thus (2.14) together with (2.15) implies

$$
f^{(k)}(z)=B \frac{\left(z-\alpha_{1}\right)^{2 m_{1}-k}\left(z-\alpha_{2}\right)^{2 m_{2}-k} \cdots\left(z-\alpha_{s}\right)^{2 m_{s}-k} g(z)}{\left(z-\beta_{1}\right)^{2 n_{1}+k}\left(z-\beta_{2}\right)^{2 n_{2}+k} \cdots\left(z-\beta_{t}\right)^{2 n_{t}+k}} .
$$

By (2.16), we obtain

$$
\left(f f^{(k)}(z)\right)^{(d+1)}=B \frac{\left(z-\alpha_{1}\right)^{2 m_{1}-k-d-1}\left(z-\alpha_{2}\right)^{2 m_{2}-k-d-1} \cdots\left(z-\alpha_{s}\right)^{2 m_{s}-k-d-1} g_{1}(z)}{\left(z-\beta_{1}\right)^{2 n_{1}+k+d+1}\left(z-\beta_{2}\right)^{2 n_{2}+k+d+1} \cdots\left(z-\beta_{t}\right)^{2 n_{t}+k+d+1}},
$$

where $\operatorname{deg}\left(g_{1}(z)\right) \leq(k+d+1)(s+t-1)$.

Next, we discuss the following two cases.

Case 1. If $f f^{(k)}-p(z)$ has only one zero $z_{0}$, then let

$$
f^{(k)}(z)-p(z)=C \frac{\left(z-z_{0}\right)^{l}}{\left(z-\beta_{1}\right)^{2 n_{1}+k}\left(z-\beta_{2}\right)^{2 n_{2}+k} \cdots\left(z-\beta_{t}\right)^{2 n_{t}+k}} .
$$

Subcase 1.1. When $d \geq l$.

Combining (2.16) and (2.18), we get $d+2 n+k t=\operatorname{deg}(g(z))+2 m-k s \leq k(s+t-1)+2 m-k s$. That is, $2(m-n) \geq k+d$, and then $m>n$.

Differentiating both sides of (2.18), we have

$$
\begin{aligned}
& \left(f f^{(k)}(z)\right)^{(d+1)}-p^{(d+1)}(z) \\
& \quad=C \frac{g_{2}(z)}{\left(z-\beta_{1}\right)^{2 n_{1}+k+d+1}\left(z-\beta_{2}\right)^{2 n_{2}+k+d+1} \cdots\left(z-\beta_{t}\right)^{2 n_{t}+k+d+1}},
\end{aligned}
$$

where $\operatorname{deg}\left(g_{2}(z)\right) \leq t(d+1)+l-d-1$.

By (2.17) and (2.19), we know $2 m-(k+d+1) s \leq \operatorname{deg}\left(g_{2}(z)\right) \leq t(d+1)+l-d-1$. Thus $2 m-(k+d+1) s-t(d+1) \leq l-d-1$. Since $2 m-(k+d+1) s-t(d+1) \geq 2 m-(k+d+$ 1) $\frac{m}{k / 2+d+1}-(d+1) \frac{n}{k / 2+d+1}>0$, then $0<l-d-1$, which contradicts with $d \geq l$.

Subcase 1.2. When $d<l$.

Differentiating both sides of (2.18), we have

$$
\begin{aligned}
& \left(f^{(k)}(z)\right)^{(d+1)}-p^{(d+1)}(z) \\
& \quad=D \frac{\left(z-z_{0}\right)^{l-d-1} g_{3}(z)}{\left(z-\beta_{1}\right)^{2 n_{1}+k+d+1}\left(z-\beta_{2}\right)^{2 n_{2}+k+d+1} \cdots\left(z-\beta_{t}\right)^{2 n_{t}+k+d+1}},
\end{aligned}
$$

where $g_{3}(z)=(l-2 n-k t) \cdots(l-2 n-k t-d) z^{t(d+1)}+\cdots+d_{1} z+d_{0}$, where $d_{i}(i=0,1)$ are constants and $\operatorname{deg}\left(g_{3}(z)\right) \leq t(d+1)$.

Differentiating both sides of (2.18) step by step for $d$ times, we have $z_{0}$ is a zero of $\left(f f^{(k)}(z)\right)^{(d)}-p^{(d)}(z)$, as $p^{(d)}(z)=a_{d} \neq 0$ and the multiplicity of all the zeros of $f(z)$ is at least $\frac{k}{2}+d+1$, thus $\alpha_{i} \neq z_{0}(i=1,2, \ldots, s)$. When $p(z)$ is a constant, from (2.18) we can also get $\alpha_{i} \neq z_{0}(i=1,2, \ldots, s)$.

Here, we discuss three subcases as follows.

Subcase 1.2.1. When $l<2 n+k t+d$.

Combining (2.16) and (2.18), we get $d+2 n+k t=\operatorname{deg}(g(z))+2 m-k s \leq k(s+t-1)+2 m-k s$. That is, $2(m-n) \geq k+d$, and then $m>n$. 
Since $\alpha_{i} \neq z_{0}(i=1,2, \ldots, s)$, by (2.17) and (2.20), we have $t(d+1) \geq \operatorname{deg}\left(g_{3}(z)\right) \geq 2 m-(k+$ $d+1) s$. Thus $2 m \leq(k+d+1) s+t(d+1) \leq(k+d+1) \frac{m}{k / 2+d+1}+(d+1) \frac{n}{k / 2+d+1}<2 m$, which is impossible.

Subcase 1.2.2. When $l=2 n+k t+d$.

If $m>n$, by a similar discussion to Subcase 1.2.1, we can get a contradiction. Thus $m \leq n$. Since $\alpha_{i} \neq z_{0}(i=1,2, \ldots, s)$, by $(2.17)$ and (2.20), we have $l-d-1 \leq \operatorname{deg}\left(g_{1}(z)\right) \leq(k+d+$ 1) $(s+t-1)$, since $l=2 n+k t+d$, thus $2 n+k t+d-d-1 \leq(k+d+1)(s+t-1)$. Then $2 n \leq(k+d+1) s+(d+1) t-(k+d)<(k+d+1) \frac{m}{k / 2+d+1}+(d+1) \frac{n}{k / 2+d+1} \leq 2 n$, which is impossible.

Subcase 1.2.3. When $l>2 n+k t+d$.

By (2.16) and (2.18), we get $l=\operatorname{deg}(g(z))+2 m-k s \leq k(s+t-1)+2 m-k s=2 m+k t-k$. If $m>n$, by a similar discussion to Subcase 1.2.1, we get a contradiction. Thus $m \leq n$.

Case 2. If $f^{(k)}-p(z)$ has no zero. Then $l=0$ in (2.18), by a similar discussion to Subcase 1.1, we get a contradiction.

By Case 1 and Case 2, we get $f f^{(k)}-p(z)$ has at least two distinct zeros.

\section{Proof of Theorem 1.1}

For any point $z_{0}$ in $D$, either $p\left(z_{0}\right)=0$ or $p\left(z_{0}\right) \neq 0$.

Case 1. When $p\left(z_{0}\right)=0$. We may assume $z_{0}=0$. Then $p(z)=a_{d} z^{d}+a_{d+1} z^{d+1}+\cdots=z^{d} h(z)$, where $a_{d}(\neq 0), a_{d+1}, \ldots$ are constants, $d \geq 1, h\left(z_{0}\right) \neq 0$, without loss of generality, let $h\left(z_{0}\right)=$ $a_{d}$, where $h(z)$ is a holomorphic function.

Let $\mathcal{F}_{1}=\left\{F_{j} \mid F_{j}=\frac{f_{j}}{z^{d / 2}}, f_{j} \in \mathcal{F}\right\}$. If $\mathcal{F}_{1}$ is not normal at 0 , then by Lemma 2.1, there exists a sequence of complex numbers $z_{j} \rightarrow 0$, a sequence of positive numbers $\rho_{j} \rightarrow 0$ and a sequence of functions $F_{j} \in \mathcal{F}_{1}$ such that $G_{j}(\xi)=\rho_{j}^{-\frac{k}{2}} F_{j}\left(z_{j}+\rho_{j} \xi\right) \rightarrow G(\xi)$ spherically locally uniformly in $\mathbb{C}$, where $G(\xi)$ is a nonconstant meromorphic function in $\mathbb{C}$, and the multiplicity of the zeros and poles of $G(\xi)$ is at least $\max \left\{\frac{k}{2}+d+1, k+d\right\}$. Here, we discuss two cases as follows.

Case 1.1. There exists a subsequence of $\frac{z_{j}}{\rho_{j}}$, we may denote it as $\frac{z_{j}}{\rho_{j}}$ such that $\frac{z_{j}}{\rho_{j}} \rightarrow c, c$ is a finite complex number. Then

$$
\phi_{j}(\xi)=\frac{f_{j}\left(\rho_{j} \xi\right)}{\rho_{j}^{\frac{d+k}{2}}}=\frac{\left(\rho_{j} \xi\right)^{\frac{d}{2}} F_{j}\left(z_{j}+\rho_{j}\left(\xi-\frac{z_{j}}{\rho_{j}}\right)\right)}{\rho_{j}^{\frac{d}{2}} \rho_{j}^{\frac{k}{2}}} \rightarrow \xi^{\frac{d}{2}} G(\xi-c)=H(\xi)
$$

spherically locally uniformly in $\mathbb{C}$, so

$$
\phi_{j}(\xi) \phi_{j}^{(k)}(\xi)-\frac{p\left(\rho_{j} \xi\right)}{\rho_{j}^{d}}=\frac{f_{j}\left(\rho_{j} \xi\right) f_{j}^{(k)}\left(\rho_{j} \xi\right)-p\left(\rho_{j} \xi\right)}{\rho_{j}^{d}} \rightarrow H(\xi) H^{(k)}(\xi)-a_{d} \xi^{d}
$$

spherically locally uniformly in $\mathbb{C}$.

Since $\forall f \in \mathcal{F}$, the multiplicity of whose zeros and poles is at least $\max \left\{\frac{k}{2}+d+1, k+\right.$ $d\}$, then the multiplicity of all zeros and poles of $H$ is at least $\max \left\{\frac{k}{2}+d+1, k+d\right\}$, by Lemmas 2.5-2.7, we get $H(\xi) H^{(k)}(\xi)-a_{d} \xi^{d} \not \equiv 0$, and $H(\xi) H^{(k)}(\xi)-a_{d} \xi^{d}$ has at least two distinct zeros.

Suppose $\xi_{0}, \xi_{0}^{*}$ are two distinct zeros of $H(\xi) H^{(k)}(\xi)-a_{d} \xi^{d}$. We may choose a proper $\sigma>0$ such that $D\left(\xi_{0}, \sigma\right) \cap D\left(\xi_{0}^{*}, \sigma\right)=\emptyset$, where $D\left(\xi_{0}, \sigma\right)=\left\{\xi|| \xi-\xi_{0} \mid<\sigma\right\}, D\left(\xi_{0}^{*}, \sigma\right)=\{\xi \| \xi-$ $\left.\xi_{0}^{*} \mid<\sigma\right\}$. 
By Hurwitz's theorem, there exists a subsequence of $f_{j}\left(\rho_{j} \xi\right) f_{j}^{(k)}\left(\rho_{j} \xi\right)-p\left(\rho_{j} \xi\right)$, we may still denote it as $f_{j}\left(\rho_{j} \xi\right) f_{j}^{(k)}\left(\rho_{j} \xi\right)-p\left(\rho_{j} \xi\right)$, then there exist points $\xi_{j} \in D\left(\xi_{0}, \sigma\right)$ and points $\xi_{j}^{*} \in$ $D\left(\xi_{0}^{*}, \sigma\right)$ such that for sufficiently large $j, f_{j}\left(\rho_{j} \xi_{j}\right) f_{j}^{(k)}\left(\rho_{j} \xi_{j}\right)-p\left(\rho_{j} \xi_{j}\right)=0, f_{j}\left(\rho_{j} \xi_{j}^{*}\right) f_{j}^{(k)}\left(\rho_{j} \xi_{j}^{*}\right)-$ $p\left(\rho_{j} \xi_{j}^{*}\right)=0$.

Since $f_{j} f_{j}^{(k)}$ and $g_{j} g_{j}^{(k)}$ share $p(z)$ in $D$, it follows that for any positive integer $m$, $f_{m}\left(\rho_{j} \xi_{j}\right) f_{m}^{(k)}\left(\rho_{j} \xi_{j}\right)-p\left(\rho_{j} \xi_{j}\right)=0, f_{m}\left(\rho_{j} \xi_{j}^{*}\right) f_{m}^{(k)}\left(\rho_{j} \xi_{j}^{*}\right)-p\left(\rho_{j} \xi_{j}^{*}\right)=0$.

Fix $m$, let $j \rightarrow \infty$ and note $\rho_{j} \xi_{j} \rightarrow 0, \rho_{j} \xi_{j}^{*} \rightarrow 0$, we obtain $f_{m}(0) f_{m}^{(k)}(0)-p(0)=0$.

Since the zeros of $f_{m}(0) f_{m}^{(k)}(0)-p(0)$ have no accumulation points, in fact when $j$ is large enough, we have $\rho_{j} \xi_{j}=\rho_{j} \xi_{j}^{*}=0$. Thus, when $j$ is large enough, $\xi_{0}=\xi_{0}^{*}=0$, which contradicts with $D\left(\xi_{0}, \sigma\right) \cap D\left(\xi_{0}^{*}, \sigma\right)=\emptyset$. Thus, $\mathcal{F}_{1}$ is normal at 0 .

Case 1.2. There exists a subsequence of $\frac{z_{j}}{\rho_{j}}$, we may denote it as $\frac{z_{j}}{\rho_{j}}$ such that $\frac{z_{j}}{\rho_{j}} \rightarrow \infty$. Then

$$
\begin{aligned}
f_{j}\left(z_{j}+\rho_{j} \xi\right) f_{j}^{(k)}\left(z_{j}+\rho_{j} \xi\right)= & \left(z_{j}+\rho_{j} \xi\right)^{\frac{d}{2}} F_{j}\left(z_{j}+\rho_{j} \xi\right)\left[\left(z_{j}+\rho_{j} \xi\right)^{\frac{d}{2}}\left(F_{j}\left(z_{j}+\rho_{j} \xi\right)\right)^{(k)}\right. \\
& \left.+\sum_{i=1}^{k} c_{i}\left(z_{j}+\rho_{j} \xi\right)^{\frac{d}{2}-i}\left(F_{j}\left(z_{j}+\rho_{j} \xi\right)\right)^{(k-i)}\right] \\
= & \left(z_{j}+\rho_{j} \xi\right)^{d} G_{j}(\xi) G_{j}^{(k)}(\xi)+\sum_{i=1}^{k} c_{i}\left(z_{j}+\rho_{j} \xi\right)^{d-i} \rho_{j}^{i} G_{j}(\xi) G_{j}^{(k-i)}(\xi),
\end{aligned}
$$

where $c_{i}=\frac{d}{2}\left(\frac{d}{2}-1\right) \cdots\left(\frac{d}{2}-i+1\right) C_{d / 2}^{i}$ when $\frac{d}{2} \geq i$, and $c_{i}=0$ when $\frac{d}{2}<i$.

Thus, we have

$$
\begin{aligned}
& \frac{a_{d} f_{j}\left(z_{j}+\rho_{j} \xi\right) f_{j}^{(k)}\left(z_{j}+\rho_{j} \xi\right)}{p\left(z_{j}+\rho_{j} \xi\right)}-a_{d} \\
& =\left(G_{j}(\xi) G_{j}^{(k)}(\xi)+\sum_{i=1}^{k} c_{i} \frac{G_{j}(\xi) G_{j}^{(k-i)}(\xi)}{\left(\frac{z_{j}}{\rho_{j}}+\xi\right)^{i}}\right) \frac{a_{d}}{h\left(z_{j}+\rho_{j} \xi\right)}-a_{d} \\
& \quad \rightarrow G(\xi) G^{(k)}(\xi)-a_{d},
\end{aligned}
$$

spherically locally uniformly in $\mathbb{C}-\{\xi \mid G(\xi)=\infty\}$.

Since the multiplicity of all zeros and poles of $G$ is at least $\max \left\{\frac{k}{2}+d+1, k+d\right\}$ and by Lemmas 2.5-2.7, we have $G(\xi) G^{(k)}(\xi)-a_{d} \not \equiv 0$, and $G(\xi) G^{(k)}(\xi)-a_{d}$ has at least two distinct zeros.

Suppose $\xi_{1}, \xi_{1}^{*}$ are two distinct zeros of $G(\xi) G^{(k)}(\xi)-a_{d}$. We may choose a proper $\delta>0$ such that $D\left(\xi_{1}, \delta\right) \cap D\left(\xi_{1}^{*}, \delta\right)=\emptyset$, where $D\left(\xi_{1}, \delta\right)=\left\{\xi|| \xi-\xi_{1} \mid<\sigma\right\}, D\left(\xi_{1}^{*}, \delta\right)=\left\{\xi|| \xi-\xi_{1}^{*} \mid<\delta\right\}$.

By Hurwitz's theorem, there exists a subsequence of $a_{d} f_{j}\left(z_{j}+\rho_{j} \xi\right) f_{j}^{(k)}\left(z_{j}+\rho_{j} \xi\right)-a_{d} p\left(z_{j}+\right.$ $\left.\rho_{j} \xi\right)$, we may still denote it as $a_{d} f_{j}\left(z_{j}+\rho_{j} \xi\right) f_{j}^{(k)}\left(z_{j}+\rho_{j} \xi\right)-a_{d} p\left(z_{j}+\rho_{j} \xi\right)$, then there exist points $\xi_{j} \in D\left(\xi_{1}, \delta\right)$ and points $\xi_{j}^{*} \in D\left(\xi_{1}^{*}, \delta\right)$ such that for sufficiently large $j, a_{d} f_{j}\left(z_{j}+\rho_{j} \xi\right) f_{j}^{(k)}\left(z_{j}+\right.$ $\left.\rho_{j} \xi\right)-a_{d} p\left(z_{j}+\rho_{j} \xi\right)=0, a_{d} f_{j}\left(z_{j}+\rho_{j} \xi\right) f_{j}^{(k)}\left(z_{j}+\rho_{j} \xi\right)-a_{d} p\left(z_{j}+\rho_{j} \xi\right)=0$.

Similar to the proof of Case 1.1, we get a contradiction. Then $\mathcal{F}_{1}$ is normal at 0 .

By Case 1.1 and Case 1.2, we know $\mathcal{F}_{1}$ is normal at 0 . Hence there exists $\triangle_{\rho}=\{z:|z|<\rho\}$ and a subsequence of $F_{j k}$ of $F_{j}$ such that $F_{j k}$ converges spherically locally uniformly to a meromorphic function $F(z)$ or $\infty(k \rightarrow \infty)$ in $\triangle_{\rho}$.

Here, we discuss the following two cases. 
Case i. When $k$ is large enough, $f_{j k} \neq 0$. Then $F(0)=\infty$. Thus, for $\forall$ constant $R>0$, $\exists \sigma \in(0, \rho)$, we have $|F(z)|>R$ when $z \in \triangle_{\rho}$. Thus, for sufficiently large $k,\left|F_{j k}(z)\right|>\frac{R}{2}, \frac{1}{f_{j k}}$ is a holomorphic function in $\triangle_{\rho}$. Hence when $|z|=\frac{\sigma}{2}$,

$$
\left|\frac{1}{f_{j k}}\right|=\left|\frac{1}{F_{j k} z^{d / 2}}\right| \leq \frac{2^{d / 2+1}}{R \sigma^{d / 2}}
$$

By the maximum principle and Montel's theorem, $\mathcal{F}$ is normal at $z=0$.

Case ii. There exists a subsequence of $f_{j k}$, we may still denote it as $f_{j k}$, such that $f_{j k}(0)=0$. Since $\forall f \in \mathcal{F}$, the multiplicity of whose zeros is at least $\max \left\{\frac{k}{2}+d+1, k+d\right\}$, then $F(0)=0$. Thus, there exists $0<r<\rho$ such that $F(z)$ is holomorphic in $\triangle_{r}=\{z:|z|<r\}$ and has a unique zero $z=0$ in $\triangle_{r}$. Then $F_{j k}$ converges spherically locally uniformly to a holomorphic function $F(z)$ in $\triangle_{r}, f_{j k}$ converges spherically locally uniformly to a holomorphic function $z^{\frac{d}{2}} F(z)$ in $\triangle_{r}$. Hence $\mathcal{F}$ is normal at $z=0$.

By Case i and Case ii, we obtain $\mathcal{F}$ is normal at $z=0$.

Case 2. When $p\left(z_{0}\right) \neq 0$.

Suppose that $\mathcal{F}$ is not normal at $z_{0}$. Then by Lemma 2.1, there exists a sequence of complex numbers $z_{t} \rightarrow z_{0}$, a sequence of positive numbers $\rho_{t} \rightarrow 0$ and a sequence of functions $f_{t} \in \mathcal{F}$ such that $g_{t}(\xi)=\rho_{t}^{-\frac{k}{2}} f_{t}\left(z_{t}+\rho_{t} \xi\right) \rightarrow g(\xi)$ spherically locally uniformly in $\mathbb{C}$, where $g(\xi)$ is a nonconstant meromorphic function in $\mathbb{C}$, and the multiplicity of the zeros and poles of $g(\xi)$ is at least $\max \left\{\frac{k}{2}+d+1, k+d\right\}$.

Hence by Lemmas 2.5-2.7, we have $g(\xi) g^{(k)}(\xi)-p\left(z_{0}\right) \not \equiv 0$, and $g(\xi) g^{(k)}(\xi)-p\left(z_{0}\right)$ has at least two distinct zeros. Similar to the proof of Case 1.1, we get a contradiction. Thus, $\mathcal{F}$ is normal at $z_{0}$.

Hence, $\mathcal{F}$ is normal in $D$ as $z_{0}$ is arbitrary. The proof is complete.

\section{Proof of Theorem 1.2}

Because $P(z)$ has at least one zero, we may assume, with no loss of generality, that $P(z)=$ $z^{n}+a_{n-1} z^{n-1}+\cdots+a_{q} z^{q}$, where $q \geq 1$ is a positive integer and $a_{q} \neq 0$. Suppose that $\mathcal{F}$ is not normal in $D$. Then similar to the proof of Theorem 1.1, we can get a contradiction. Hence $\mathcal{F}$ is normal in $D$. The proof is complete.

\section{Competing interests}

The authors declare that they have no competing interests.

\section{Authors' contributions}

LQ and FH performed and drafted manuscript. All authors read and approved the final manuscript.

\section{Acknowledgements}

The authors wish to thank the referees and editors for their very helpful comments and useful suggestions.

\section{Received: 7 November 2012 Accepted: 9 May 2013 Published: 6 June 2013}

\section{References}

1. Hayman, WK: Meromorphic Functions. Clarendon, Oxford (1964)

2. Schiff, J: Normal Families. Springer, Berlin (1993)

3. Yang, CC: Value Distribution Theory. Springer, Berlin (1993)

4. Hayman, WK: Research Problems in Function Theory. Athlone Press, London (1967)

5. Mues, E: Über ein problem von Hayman. Math. Z. 164, 239-259 (1979)

6. Clunie, J: On a result of Hayman. J. Lond. Math. Soc. 42, 389-392 (1967)

7. Yang, L, Yang, CC: Angular distribution of values of ff'. Sci. China Ser. A 37, 284-294 (1994)

8. Zhang, ZF, Song, GD: On the zeros of $f\left(f^{(k)}\right)^{n}-a(z)$. Chin. Ann. Math., Ser. A 19(2), 275-282 (1998)

9. Wang, YM: On the value distribution of $f^{n} f^{(k)}-c(z)$. Journal of Huaibei Coal Industry Teachers College. Natural Science Edition 26(4), 1-4 (2005). doi:10.3969/j.issn.2095-0691.2005.04.001 
10. Wang, JP: A fundamental inequality of theory of meromorphic function and its applications. Acta Math. Sin. 49(2), 443-450 (2006) (in Chinese)

11. Meng, DW, Hu, PC: Normality criteria of meromorphic functions sharing one value. J. Math. Anal. Appl. 381, 724-731 (2011)

12. Jiang, YB, Gao, ZS: Normal families of meromorphic functions sharing values or functions. J. Inequal. Appl. 2011 Article ID 72 (2011). doi:10.1186/1029-242X-2011-72

13. $\mathrm{Wu}, \mathrm{XZ}, \mathrm{Xu}, \mathrm{Y}$ : Normal families of meromorphic functions and shared values. Monatshefte Math. 165, 569-578 (2012)

14. Pang, XC, Zalcman, L: Normal families and share values. Bull. Lond. Math. Soc. 32, 325-331 (2000)

15. Hua, XH, Chuang, CT: On the conjecture of Hayman. Acta Math. Sin. New Ser. 7(2), 119-126 (1991)

16. Clunie, J: On the integral and meromorphic functions. J. Lond. Math. Soc. 37, 17-27 (1962)

doi:10.1186/1029-242X-2013-288

Cite this article as: Qiu and Hu: Normal families of meromorphic functions sharing one function. Journal of Inequalities and Applications 2013 2013:288.

Submit your manuscript to a SpringerOpen ${ }^{\circ}$ journal and benefit from:

- Convenient online submission

- Rigorous peer review

- Immediate publication on acceptance

- Open access: articles freely available online

- High visibility within the field

- Retaining the copyright to your article

Submit your next manuscript at $>$ springeropen.com 